\section{Einladung nach Davos}

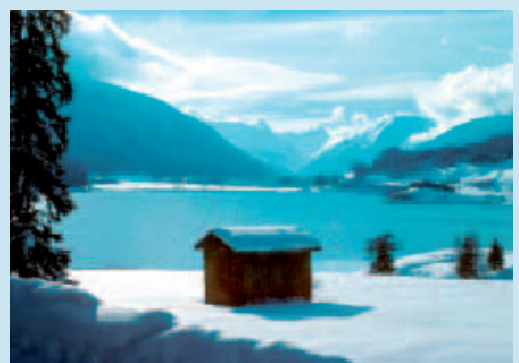

Auch im Jahr 2008 richtet das Europäische Allergie- und Asthma-Centrum Davos (EACD) wieder ein internationales Symposium mit dem Titel "Allergologie“ aus. Es wird vom 4. bis 6. April 2008 an der Hochgebirgsklinik Davos stattfinden. Vorsitzender des Programmkomitees ist Prof. Dr. J. Christian Virchow, Rostock. Das Themenspektrum der Veranstaltung deckt viele Bereiche der praktischen Allergologie ab, der Schwerpunkt liegt auf pneumologischen Krankheitsbildern. Da die Teilnehmerzahl begrenzt ist, wird um frühzeitige Anmeldung gebeten.

Für Informationen und Anmeldungen wenden Sie sich bitte an EACD c/o Hochgebirgsklinik Davos, Frau Heike Virchow, Tel.: (oo 41-81) 41731 37, Fax: (oo 41-81) 417 30 34, E-Mail: heike.virchow@hgk.ch. red

\section{Neue COPD-Leitlinie}

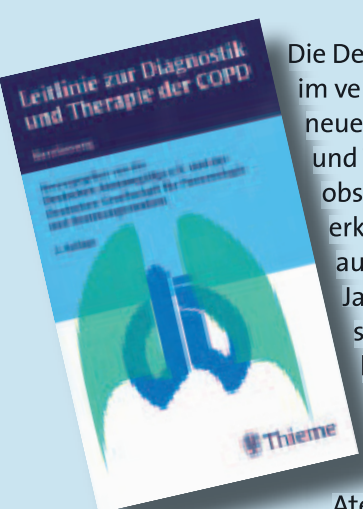

Die Deutsche Atemwegsliga hat im vergangenen August eine neue Leitlinie zur Diagnostik und Therapie chronischer obstruktiver Atemwegserkrankungen (COPD) herausgegeben., In den letzten Jahren hat sich unser Verständnis der COPD grundlegend geändert", erklärte dazu Prof. Dr. Hein rich Worth, Fürth, Vorsitzender der Deutschen Atemwegsliga. Wurde die COPD bislang als reine Lungenerkrankung eingestuft, zeigen neuere Untersuchungen, dass die Erkrankung mit einer Entzündungsreaktion einhergeht, die den gesamten Körper beeinflusst. Die neuen Erkenntnisse haben eine Aktualisierung der COPD-Leitlinie erforderlich gemacht. Neben der Vollversion der Leitlinie gibt es auch eine komprimierte Fassung für die Kitteltasche. Beide stehen unter www.atemwegsliga auf der Homepage der Atemwegsliga zum Download bereit, gedruckte Versionen können dort ebenfalls bestellt werden.

\title{
Gratulation an einen Brückenbauer
}

P rof. Dr. med. Thomas Fuchs beging am 21. September 2007 seinen 60. Geburtstag. Die Deutsche Gesellschaft für Allergologie und klinische Immunologie (DGAKI) gratuliert Prof. Fuchs hierzu sehr herzlich.

Herr Fuchs wurde am 21. September 1947 in Göttingen geboren. Das Medizinstudium absolvierte er von 1968 bis 1975 in München und Göttingen. Die Weiterbildung zum Hautarzt und Allergologen erfolgte von 1977 bis 1981 an der Universitäts-Hautklinik Göttingen bei Prof. Dr. Helmut Ippen. Anschließend war Herr Fuchs von 1981 bis 1983 Assistent in der Abteilung für Allergologie in den Städtischen Kliniken Stuttgart-Bad Cannstadt bei Prof. Dr. Dieter Kleinhans, bevor er 1983 als Oberarzt an die Universitäts-Hautklinik Göttingen zurückkehrte. 1986 wurde er Leiter des Funktionsbereiches Allergologie. 1993 erfolgte die Habilitation mit dem Thema „Die Gummiallergie“. Im Jahr 1998 wurde er zum außerplanmäßigen Professor für Dermatologie und Venerologie ernannt. Wissenschaftlich hat sich Prof. Fuchs insbesondere mit Kontaktallergien, speziell mit berufsdermatologischen Fragesellungen beschäftigt.

Der DGAKI ist Herr Fuchs vor allem durch seine Zeit als Präsident des Ärzteverbandes Deutscher Allergologen

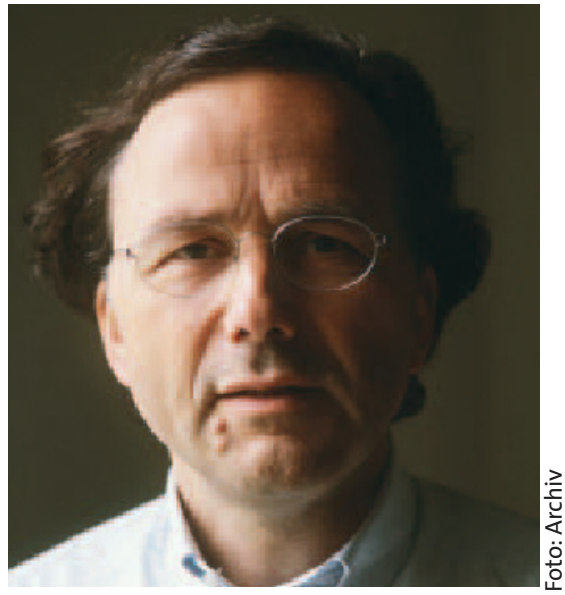

Ad multos annos: Prof. Dr. Thomas Fuchs, Göttingen

in den Jahren 2000 bis 2004 sowie durch seine Tätigkeit im Beirat der DGAKI und des Allergo Journals verbunden. Herr Fuchs war an der Ausrichtung des gemeinsamen Allergiekongresses 2004 in Aachen beteiligt und wird den dritten gemeinsamen Kongress in Erfurt zusammen mit Frau Priv.-Doz. Dr. Kirsten Jung organisieren. Herr Fuchs hat wesentlich dazu beigetragen, eine Brücke zwischen den berufspolitischen und wissenschaftlichen nationalen Fachgesellschaften zu schlagen.

Prof. Dr. Gerhard Schultze-Werninghaus, Bochum

\section{Ausgezeichneter T-Zell-Forscher}

D er Allergopharma Award ist in $\mathrm{Zu}$ sammenarbeit mit der European Academy of Allergology and Clinical Immunology geschaffen worden, um hervorragende Forschungen jüngerer Mitglieder der Akademie auf dem Gebiet der Mechanismen der allergischen Entzündung sowie der spezifischen Immuntherapie zu würdigen. Der diesjährige Award wurde während des XXVI. Kongresses der Akademie in Göteborg, Schweden, an Dr. Carsten SchmidtWeber vom Schweizerischen Institut für Allergie- und Asthmaforschung in Davos verliehen.
Es gibt inzwischen verstärkt Hinweise darauf, dass regulatorische T-Zellen eine wichtige Rolle bei der Kontrolle der Immunantwort auf Allergene spielen. Dr. Schmidt-Weber ist aufgrund seiner Arbeit über die molekularen Mechanismen, die der Induktion von FOXP3 - eines wesentlichen Transkriptionsfaktors in T-Lymphozyten - zugrunde liegen, für den Allergopharma Award ausgewählt worden. Seine Forschung hat neue Einblicke erbracht in die Induktionsmechanismen des Transkriptionsfaktors sowie die Rolle, die er für regulatorische T-Zellen spielt. red 